\title{
Prognostic Value of Biochemical Response Models for Primary Biliary Cholangitis and the Additional Role of the Neutrophil-to-Lymphocyte Ratio
}

\author{
Jeong-Ju Yoo ${ }^{1}$, Eun Ju Cho ${ }^{2}$, Bora Lee ${ }^{3}$, Sang Gyune $\mathrm{Kim}^{1}$, Young Seok Kim ${ }^{1}$, Yun Bin Lee ${ }^{2}$, Jeong-Hoon Lee ${ }^{2}$, Su Jong Yu², \\ Yoon Jun Kim², and Jung-Hwan Yoon ${ }^{2}$ \\ ${ }^{1}$ Department of Gastroenterology and Hepatology, Soonchunhyang University School of Medicine, ${ }^{2}$ Department of Internal Medicine and Liver \\ Research Institute, Seoul National University College of Medicine, ${ }^{3}$ Department of Statistics, Graduate School, Chung-Ang University, Seoul, \\ Korea
}

See editorial on page 613.

Background/Aims: Recently reported prognostic models for primary biliary cholangitis (PBC) have been shown to be effective in Western populations but have not been wellvalidated in Asian patients. This study aimed to compare the performance of prognostic models in Korean patients and to investigate whether inflammation-based scores can further help in prognosis prediction. Methods: This study included 271 consecutive patients diagnosed with PBC in Korea. The following prognostic models were evaluated: the Barcelona model, the Paris-l/II model, the Rotterdam criteria, the GLOBE score and the UK-PBC score. The neutrophil-to-lymphocyte ratio (NLR) was analyzed with reference to its association with prognosis. Results: For predicting liver transplant or death at the 5-year and 10-year follow-up examinations, the UK-PBC score (areas under the receiver operating characteristic curve [AUCs], 0.88 and 0.82) and GLOBE score (AUCs, 0.85 and 0.83 ) were significantly more accurate in predicting prognosis than the other scoring systems (all $p<0.05$ ). There was no significant difference between the performance of the UK-PBC and GLOBE scores. In addition to the prognostic models, a high NLR (>2.46) at baseline was an independent predictor of reduced transplant-free survival in the multivariate analysis (adjusted hazard ratio, 3.74; $p<0.01$ ). When the NLR was applied to the prognostic models, it significantly differentiated the prognosis of patients. Conclusions: The UK-PBC and GLOBE scores showed good prognostic performance in Korean patients with PBC. In addition, a high NLR was associated with a poorer prognosis. Including the NLR in prognostic models may further help to stratify patients with PBC. (Gut Liver 2018;12:714-721)

Key Words: Liver cirrhosis, biliary; Prognosis; Neutrophil-tolymphocyte ratio

\section{INTRODUCTION}

Primary biliary cholangitis $(\mathrm{PBC})$ is an autoimmune liver disease characterized by chronic cholestasis. ${ }^{1}$ Without appropriate treatment, it can proceed to liver cirrhosis and is one of the major causes of liver transplantation (LT) in Western countries. ${ }^{2}$ Standard treatment for PBC is ursodeoxycholic acid (UDCA), but approximately $30 \%$ of patients with $\mathrm{PBC}$ do not respond to this treatment, resulting in poor prognosis and the need for LT or, in the worst-case scenario, death. ${ }^{2}$ Therefore, it is important to identify a biomarker that can predict patients who do not respond to UDCA therapy or whose prognosis is poor. In order to predict the prognosis of patients with $\mathrm{PBC}$, various criteria such as the Paris-I, Paris-II, and Barcelona criteria have been developed. ${ }^{3-5}$ These criteria serve to classify PBC patients as responders or non-responders after 1 or 2 years of UDCA therapy. Recently, the UK-PBC risk score and the GLOBE score have been developed, allowing more accurate prediction of the prognosis of PBC patients than previous scoring systems. ${ }^{6,7}$ However, it is not yet clear whether these scoring systems are acceptable for Asian patients, as the UK-PBC score was developed for British patients and the GLOBE score for North American and European subjects. There is a possibility that the prognosis differs geographically, as the prevalence of $\mathrm{PBC}$ varies in different countries.

Correspondence to: Eun Ju Cho

Department of Internal Medicine and Liver Research Institute, Seoul National University College of Medicine, 101 Daehak-ro, Jongno-gu, Seoul 03080, Korea

Tel: +82-2-2072-2228, Fax: +82-2-743-6701, E-mail: creatioex@gmail.com

Received on June 12, 2018. Revised on July 21, 2018. Accepted on July 27, 2018. Published online October 29, 2018

pISSN 1976-2283 eISSN 2005-1212 https://doi.org/10.5009/gnl18271

@ This is an Open Access article distributed under the terms of the Creative Commons Attribution Non-Commercial License (http://creativecommons.org/licenses/by-nc/4.0) which permits unrestricted non-commercial use, distribution, and reproduction in any medium, provided the original work is properly cited. 
Inflammation is a principal feature of chronic liver disease and predisposition to liver cirrhosis. Therefore, inflammationbased scores may be potentially useful to predict disease progression. The neutrophil-to-lymphocyte ratio (NLR) and the platelet-to-lymphocyte ratio (PLR) have recently been shown to be prognostic markers for various inflammatory diseases and cancers, but associations with PBC have not yet been published..$^{8,9}$

The aim of this study was to compare and validate the prognostic performance of various PBC prognostic models including the UK-PBC and GLOBE scores in Korean patients, and to evaluate the impact of inflammation-based scores on $\mathrm{PBC}$ prognosis, represented by NLR and PLR.

\section{MATERIALS AND METHODS}

\section{Patients}

Between January 2000 and December 2016, we collected the data from consecutive PBC patients data obtained during routine clinical care in two tertiary hospitals (Seoul National University Hospital and Soonchunhyang University Bucheon Hospital). PBC was diagnosed when two or more of the following three criteria were met: (1) cholestatic pattern of liver function abnormality; (2) positivity for anti-mitochondrial antibodies (AMA); and (3) consistency with PBC in liver biopsy. ${ }^{2}$ For the current study, only patients with complete laboratory values after 1 year of UDCA therapy were included. Exclusion criteria were as follows: evidence of hepatitis B or hepatitis C virus, alcoholic liver disease, or primary sclerosing cholangitis or other liver disease including Wilson's disease or hemochromatosis. Finally, we retrospectively included 271 patients who met the inclusion criteria (Supplementary Fig. 1). In these patients, we compared the following six different prognostic models; Barcelona criteria, ${ }^{3}$ Paris-I criteria, ${ }^{4}$ Paris-II criteria, ${ }^{5}$ Rotterdam, ${ }^{10}$ GLOBE score, ${ }^{6}$ and UK-PBC score. ${ }^{7}$ Clinical and laboratory records of all patients were reviewed at baseline, and at 1-year, 5-year and 10-year follow-up examinations. The reference ranges for laboratory values of each hospital are described in Supplementary Table 1.

The study protocol was approved by the Institutional Review Board of each hospital, and the informed consent was waived. The study protocol also conformed to the ethical guidelines of the World Medical Association Declaration of Helsinki.

\section{Definitions}

Transplantation-free survival (TFS) was defined as the time from PBC diagnosis to LT or death. Decompensated liver disease was defined if any of the following were found during baseline or the observational period: ascites, variceal hemorrhage, hepatic encephalopathy, or jaundice more than three times that of the upper limit of normal (ULN). ${ }^{11,12}$

NLR was measured by dividing the absolute neutrophil count by the lymphocyte count, and PLR was defined as the absolute platelet count divided by the absolute lymphocyte count in a complete blood count test. ${ }^{13}$

\section{Statistical analysis}

Frequencies and percentages were used for descriptive statistics. Statistical differences between the groups were investigated using the chi-square test or Fisher exact test for categorical variables and the Student t-test or Mann-Whitney U-test for continuous variables. Survival rate was estimated using the

Table 1. Characteristics and Outcomes of Enrolled Patients

\begin{tabular}{|c|c|c|c|c|}
\hline Variable & $\begin{array}{c}\text { Total } \\
(\mathrm{n}=271)\end{array}$ & $\begin{array}{l}\text { Good prognosis } \\
\qquad(\mathrm{n}=228)\end{array}$ & $\begin{array}{l}\text { Poor prognosis } \\
\qquad(\mathrm{n}=43)\end{array}$ & p-value* \\
\hline Age, yr & $56.4 \pm 12.0$ & $55.2 \pm 11.0$ & $62.8 \pm 15.0$ & $<0.01$ \\
\hline Female sex & $236(87.1)$ & $203(89.0)$ & $33(76.7)$ & 0.04 \\
\hline \multicolumn{5}{|l|}{ Clinical status } \\
\hline Ascites grade & & & & $<0.01$ \\
\hline None & $258(95.2)$ & $225(98.7)$ & 33 (76.7) & \\
\hline Well-controlled by diuretics & $11(4.1)$ & $2(0.9)$ & $9(20.9)$ & \\
\hline Uncontrolled by diuretics & $2(0.7)$ & $1(0.4)$ & $1(2.4)$ & \\
\hline Hepatic encephalopathy & $6(2.2)$ & $1(0.4)$ & $5(11.6)$ & $<0.01$ \\
\hline Diabetes mellitus & $31(11.4)$ & $25(11.0)$ & $6(14.0)$ & 0.60 \\
\hline Hypertension & 53 (19.6) & $45(19.7)$ & $8(18.6)$ & 0.99 \\
\hline \multicolumn{5}{|l|}{ Laboratory data } \\
\hline AMA positivity & $242(89.3)$ & $204(89.5)$ & $38(88.4)$ & 0.79 \\
\hline MELD score & $8.1 \pm 3.1$ & $7.7 \pm 2.8$ & $10.0 \pm 3.8$ & $<0.01$ \\
\hline
\end{tabular}

Data are presented as mean \pm SD or number $(\%)$. p-values were calculated by Fisher exact test for categorical variables. AMA, anti-mitochondrial antibody; MELD, Model for End-Stage Liver Disease. 


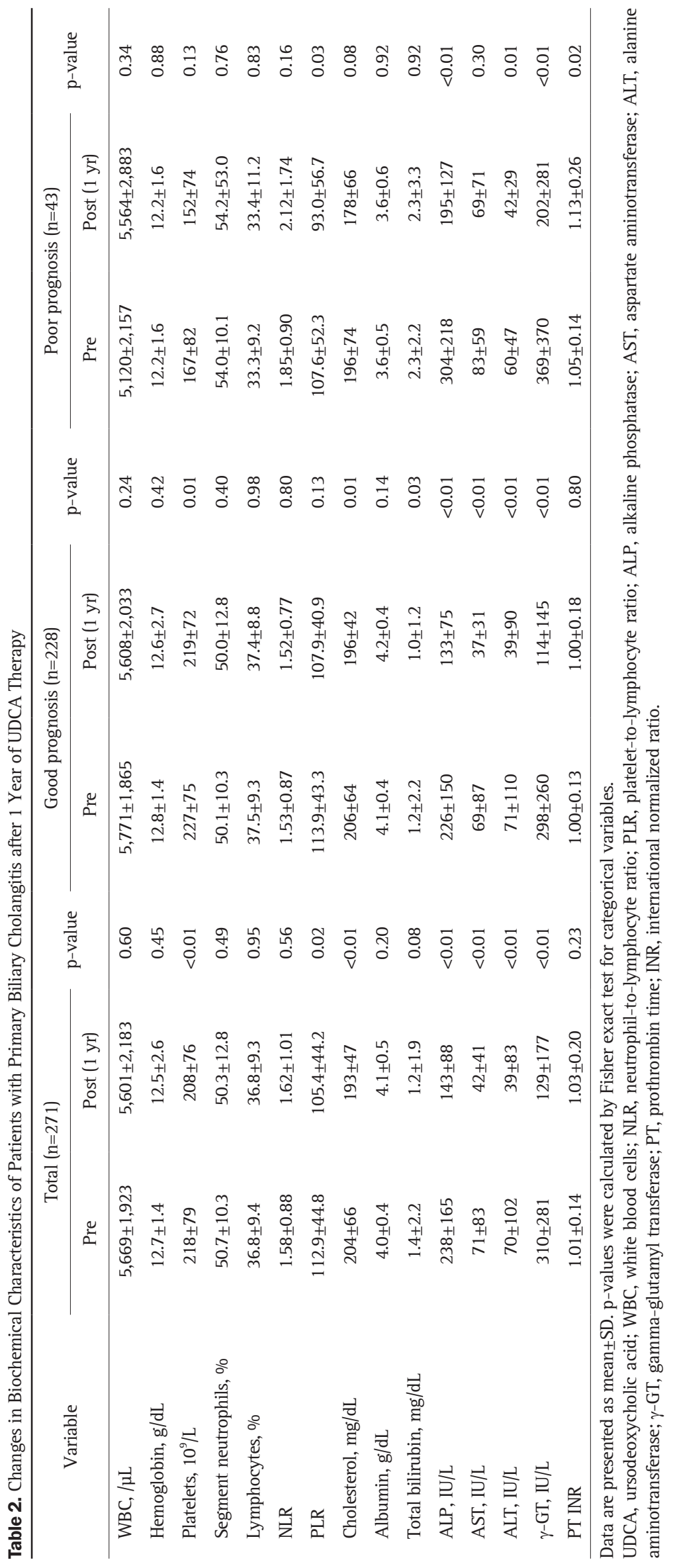



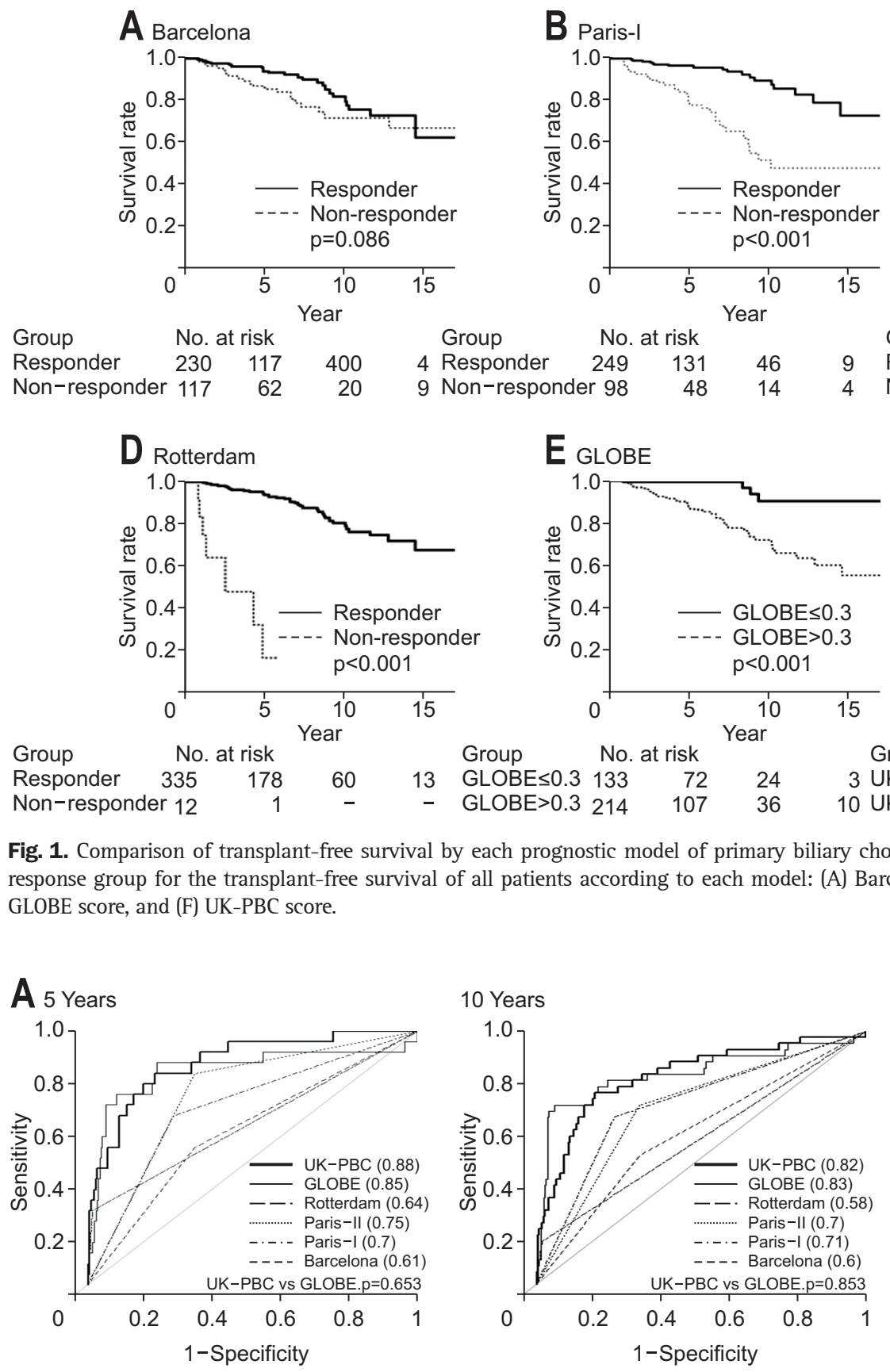

Group

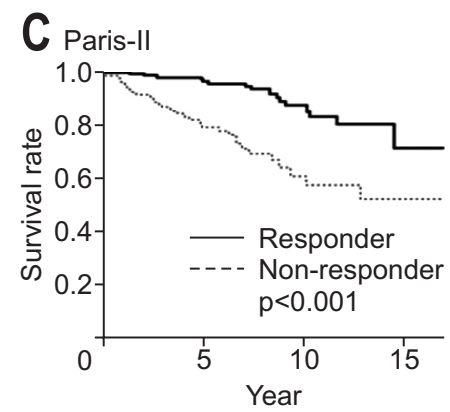

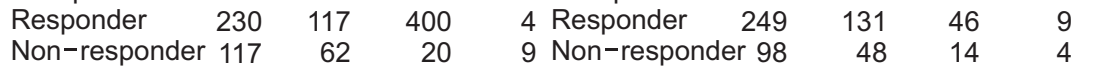

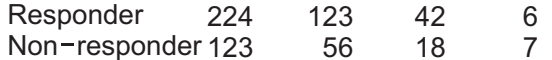
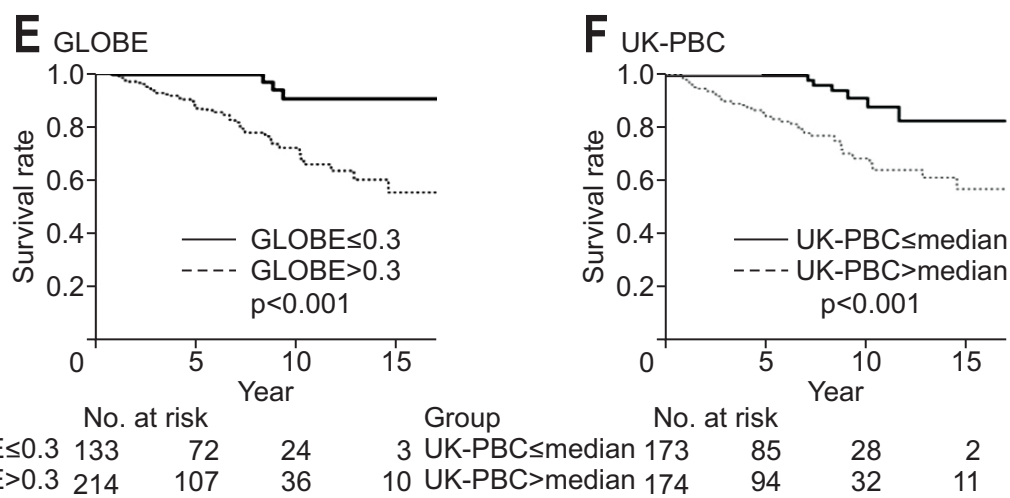

Fig. 1. Comparison of transplant-free survival by each prognostic model of primary biliary cholangitis. Kaplan-Meier survival plot stratified by response group for the transplant-free survival of all patients according to each model: (A) Barcelona, (B) Paris-I, (C) Paris-II, (D) Rotterdam, (E) GLOBE score, and (F) UK-PBC score.
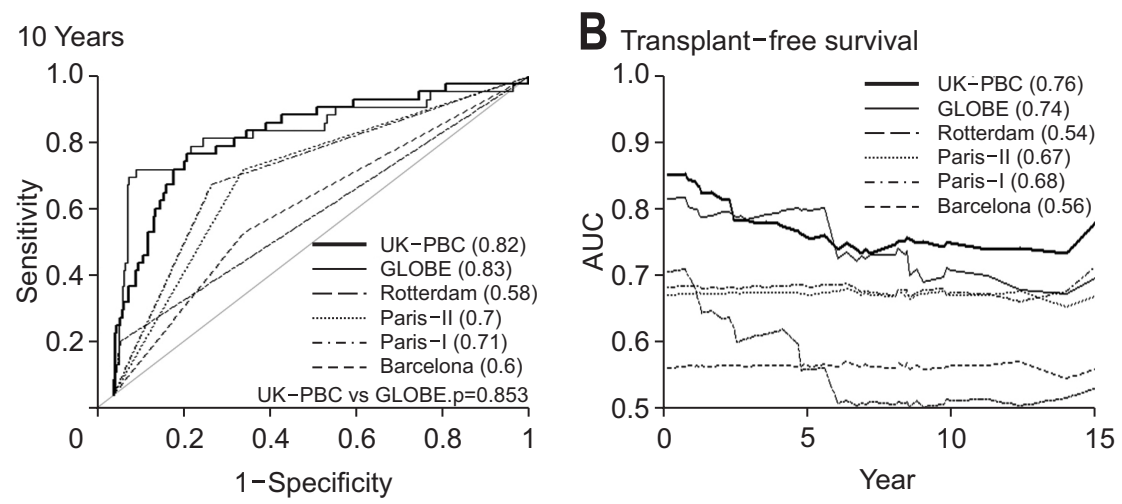

Fig. 2. The time-dependent area under the receiver operating characteristic curve (AUC) for six prognostic models of primary biliary cholangitis. (A) Predictive power of transplantation-free survival (TFS) for each model at 5-year and 10-year follow-up examinations. (B) The time-dependent AUC for predicting TFS for each model in all patients.

Kaplan-Meier method, and differences between the curves were compared using the log-rank test. The optimal cutoff value for continuous variable was determined using the "maxstat" package, a maximal chi-square method in open source software $R$. To evaluate the predictive accuracy of each prognostic model, the time-dependent receiver operating characteristic (ROC) curves for censored data and the areas under the ROC curve (AUC) were constructed according to Heagerty et al. ${ }^{14}$ All statistical analyses were performed using $\mathrm{R}$ (version 3.3.3; The $\mathrm{R}$
Foundation for Statistical Computing, Vienna, Austria) and statistical significance was defined as a $\mathrm{p}<0.05$.

\section{RESULTS}

\section{Baseline characteristics and outcomes}

The baseline characteristics of the patients enrolled in the study are reported in Table 1 . The mean age of the patients was $56.4 \pm 12.0$ years, and $87.1 \%$ (236) were female. The positive rate 


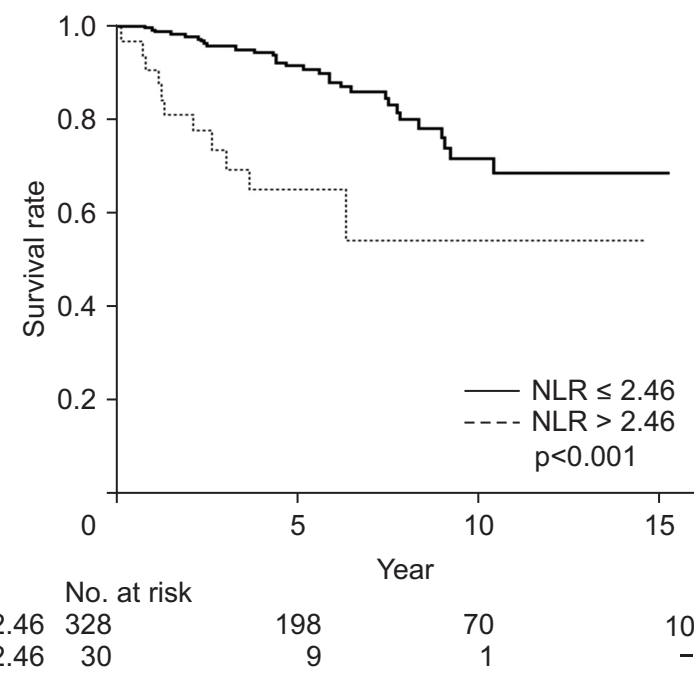

Fig. 3. Effect of the neutrophil-to-lymphocyte ratio (NLR) on prognosis in primary biliary cholangitis. Comparison of transplantation-free survival based on an NLR cutoff of 2.46 in all patients.

of AMA was 89.3\% (242) and the mean Model for End-Stage

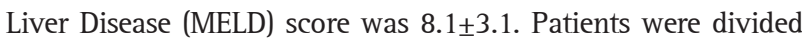
into two groups: patients with good prognosis who survivedwithout LT and patients with poor prognosis who underwent LT or died. A total of 228 patients (84.1\%) were included in the good prognosis group, and 43 patients (15.9\%) formed the poor prognosis group. The poor prognosis group comprised older patients at the time of PBC diagnosis, who had deteriorated liver function. The MELD score and NLR score were higher in patients in the poor prognosis group compared to those in the good prognosis group. Otherwise, there were no significant differences in sex and AMA positivity between the two groups.

Patients received standard UDCA treatment according to their body weight, and the mean UDCA dose was $750 \mathrm{mg}$ per day. The biochemical changes before and 1 year after UDCA treatment are described in Table 2. After UDCA treatment, levels of liver enzymes (total bilirubin, alkaline phosphatase, aspartate aminotransferase, alanine aminotransferase, gamma-glutamyl transferase) were significantly lower compared to before treatment, which applied to both the good and poor prognosis groups. Platelet counts and PLR scores also significantly decreased after 1 year, especially in the poor prognosis group.

The median observation time of the patients was 74 months (interquartile range, 36 to 109 months). During the observational period, 31 patients (11.4\%) progressed into decompensation, $33(12.2 \%)$ died (26 from liver-related causes and seven from other causes), and 10 (3.7\%) underwent LT. The overall TFS rate was $91 \%$ at 5 years, $77 \%$ at 10 years, and $69 \%$ at 15 years.

\section{Comparison of the prognostic performance for each model}

We examined whether the six models of PBC prognosis dif- ferentiated the actual outcome of the patients using the logrank test. The threshold of GLOBE score $(>0.30)$ for biochemical non-response was based on the original article. ${ }^{6}$ As there is no specific risk threshold presented for UK-PBC, patients were dichotomized according to the median value of our study cohort. As expected, all the models except for the Barcelona criteria showed significantly different parameters in patients with good outcome compared to those with poor outcomes (all $\mathrm{p}<0.001$ ) (Fig. 1). However, the Barcelona score failed to show a statistically significant difference between the two groups when applied to our patient population $(\mathrm{p}=0.086)$. Next, we compared the predictive performance power of TFS for each model at the 5-year follow-up and 10-year follow-up. When the performances of the models were compared, the UK-PBC and GLOBE scores showed significantly better discrimination, as indicated by the area-under the curves (AUCs) of 0.88 (95\% confidence interval [CI], 0.80 to 0.95 ) and 0.85 (95\% CI, 0.73 to 0.96) than the ParisI (AUC, 0.70; 95\% CI, 0.60 to 0.80), Paris-II (AUC, 0.75; 95\% CI, 0.67 to 0.84 ), Rotterdam (AUC, $0.64 ; 95 \% \mathrm{CI}, 0.54$ to 0.73 ) and Barcelona criteria (AUC, $0.61 ; 95 \% \mathrm{CI}, 0.50$ to 0.71 ) for predicting transplant or death within 5 years (all $\mathrm{p}<0.05$ ) (Fig. 2A). The difference between the UK-PBC and GLOBE scores was not statistically significant $(p=0.65)$. The performances of the UK-PBC and GLOBE scores for predicting events within 10 years were similar (AUCs, 0.82; 95\% CIs, 0.75 to 0.90 and AUCs, 0.83; 95\% CI, 0.75 to $0.92 ; p=0.86$, respectively) and significantly better than the other criteria (all $\mathrm{p}<0.05$ ). The time-dependent AUCs for the overall predictive ability of the UK-PBC and GLOBE scores were 0.76 and 0.74 , respectively, whereas the other criteria showed limited performance (all AUCs <0.7) (Fig. 2B).

\section{Association with inflammation and PBC prognosis}

Finally, we analyzed the relationship between inflammationbased scores and PBC prognosis. NLR analyzed as continuous variable was significantly associated with TFS in the univariate analysis (hazard ratio [HR], 1.43; 95\% CI, 1.15 to 1.78 ; $p=0.001$ ), whereas the PLR showed no statistical significance $(p=0.25)$. Using the maximal chi-square method, the optimal cutoff of NLR to predict prognosis was 2.46. According to the cutoff point, Kaplan-Meier estimates are shown in Fig. 3. Because of the small number of events, the median TFS could not be estimated for either group. However, patients with high NLR levels ( $>2.46)$ had a significantly higher risk of LT or death compared with patient with low NLR levels $(\leq 2.46$; HR, 4.85; 95\% CI, 2.48 to $9.51 ; \mathrm{p}<0.001)$. In addition, when NLR with a cutoff of 2.46 was applied to the six models, it significantly discriminated the prognosis of patients (Supplementary Fig. 2). When multivariate analyzes of factors affecting the prognosis of $\mathrm{PBC}$ patients were performed, a high NLR (HR, 3.74; 95\% CI, 1.53 to 9.18; $p=0.004$ ) and the GLOBE score (HR, 4.03, 95\% CI, 2.85 to 5.72, $\mathrm{p}<0.001$ ) were independent prognostic factors for shorter TFS, along with 
Table 3. Predictive Factors of Prognosis in Primary Biliary Cholangitis

\begin{tabular}{|c|c|c|c|c|c|c|}
\hline \multirow{2}{*}{ Variable } & \multicolumn{2}{|c|}{ Univariate analysis } & \multicolumn{2}{|c|}{ Multivariate analysis* } & \multicolumn{2}{|c|}{ Multivariate analysis $^{\dagger}$} \\
\hline & HR $(95 \% \mathrm{CI})$ & p-value & HR (95\% CI) & p-value & HR (95\% CI) & p-value \\
\hline Age & $1.10(1.06-1.13)$ & $<0.01$ & - & 0.06 & $1.12(1.08-1.16)$ & $<0.01$ \\
\hline Sex (male vs female) & $3.09(1.54-6.20)$ & $<0.01$ & - & 0.94 & - & 0.38 \\
\hline MELD & $1.21(1.14-1.28)$ & $<0.01$ & $1.17(1.06-1.30)$ & $<0.01$ & $1.18(1.06-1.31)$ & $<0.01$ \\
\hline NLR $(>2.46)$ & $4.84(2.31-10.10)$ & $<0.01$ & $3.74(1.53-9.18)$ & $<0.01$ & $4.20(1.67-10.55)$ & $<0.01$ \\
\hline GLOBE score & $4.39(3.24-5.96)$ & $<0.01$ & $4.03(2.85-5.72)$ & $<0.01$ & - & - \\
\hline UK-PBC score & $3.35(2.23-5.02)$ & $<0.01$ & - & - & $3.23(1.90-5.49)$ & $<0.01$ \\
\hline Barcelona & $0.57(0.31-1.08)$ & 0.09 & - & - & - & - \\
\hline Paris I & $0.23(0.12-0.44)$ & $<0.01$ & - & 0.44 & - & 0.31 \\
\hline Paris II & $0.27(0.14-0.51)$ & $<0.01$ & - & 0.31 & - & 0.25 \\
\hline Rotterdam & $0.18(0.08-0.42)$ & $<0.01$ & - & 0.17 & - & 0.56 \\
\hline
\end{tabular}

HR, hazard ratio; CI, confidence interval; MELD, Model for End-Stage Liver Disease; NLR, neutrophil-to-lymphocyte ratio.

${ }^{*}$ Model including age, sex, MELD, NLR, GLOBE score, and four response criteria; ${ }^{\dagger}$ Model including age, sex, MELD, NLR, UK-PBC score, and four response criteria.

the MELD score (Table 3, model 1). If the UK-PBC score was incorporated in the analysis instead of the GLOBE score (model 2), a high NLR was also independently associated with shorter TFS (HR, 4.20; 95\% CI, 1.67 to $10.55 ; \mathrm{p}=0.002$ ) as well as UK-PBC score (HR, 3.23; 95\% CI, 1.90 to 5.49; $\mathrm{p}<0.001$ ), MELD score and age.

\section{DISCUSSION}

Our study validated the prognostic values of six biochemical response models of PBC in Koreans, and showed that high NLR level at baseline was associated with future risk of poor outcome, independently of UDCA response. As far as we know, our investigation includes the largest number of Korean patients in a multicenter validation study.

Eight prognostic models for PBC have been published so far. ${ }^{6,715}$ Among them, we were unable to analyze the Toronto or the Mayo model because of lack of retrospective data (i.e., laboratory findings after 2-year UDCA, and presence of peripheral edema). Therefore, six prognostic models were assessed in our study. Among them, the Barcelona criteria showed lower predictive power than the other prognostic models. With regard to endpoints, the Barcelona criteria has been developed to predict overall survival, ${ }^{3}$ whereas the other scoring systems have been developed to predict TFS. Therefore, the low performance of the Barcelona criteria for the prediction of TFS is probably due to different endpoint. Also, the Barcelona criteria has shown somewhat low predictive power than the Paris or Mayo model in the previous study. ${ }^{16}$ For the recently developed GLOBE and UKPBC scores, they are more predictive than the previous models, using continuous variables instead of categorical variables. In addition, most of the other models are based on the laboratory data collected 1-year after taking UDCA, and the GLOBE and
UK-PBC scores use laboratory values from two points (baseline and 1-year after talking UDCA), which make them more precise. Due to this feature, although both models were developed for Western people, they may have high predictive power in Asians, together. ${ }^{17}$ However, because the cutoff of GLOBE score for nonresponse group was determined based on the reference population data from the Netherlands, the appropriate cutoff point in Korean patients should be evaluated in a future study.

It is now known that inflammation is one of the major pathophysiological mechanisms for the development and progression of PBC. ${ }^{18,19}$ Especially in recent years, the terms have changed from "primary biliary cirrhosis" to "primary biliary cholangitis," which, by definition, tends to focus more on the inflammation of bile ducts. ${ }^{2}$ NLR is a value that has been recently identified in relation to systemic inflammation, and has been extensively studied in cancer and cardiovascular disease. ${ }^{20-22}$ In relation to liver disease, the potential of NLR as a predictor of advanced histology or prognosis has been reported in hepatitis B virus and hepatitis C virus, and nonalcoholic fatty liver disease. ${ }^{9,23,24}$ However, with regard to $\mathrm{PBC}$, only one cohort study has reported the association of high NLR and short-term (1-year) mortality in hospitalized patients with $\mathrm{PBC}{ }^{25}$ Consistent with this, our study proved the value of NLR for the prediction of long-term prognosis.

The cutoff values of NLR differ greatly for disease entity, being 5.0 for liver cancer, and 5.7 for acute-on-chronic liver failure (ACLF)-induced decompensation. ${ }^{26}$ In ACLF-induced decompensation, NLR even showed significantly better predictability than the MELD, which thus shows potential for NLR as a promising biomarker. ${ }^{27}$ In the present study, the cutoff NLR value was about 2.4, indicating that the degree of inflammation was comparatively lower than that of cancer or ACLF.

Taken together, our findings suggest that the combination of 
pre-existing models, for example, the GLOBE or UK-PBC scores with inflammatory markers such as NLR may be more predictive for the prognosis of PBC. Indeed, one of the AMA markers, gp210, has been reported to help predict prognosis, to some extent, and to help optimize the GLOBE score in Chinese patients. ${ }^{16}$ In the case of the article in question, however, the endpoint is a complication of cirrhosis, and is therefore slightly different from our study where the endpoint is TFS. In this study, high NLR level at baseline was able to independently predict poor outcome, even after adjusting for UK-PBC or GLOBE scores. Furthermore, in the subgroup with a predicted favorable outcome according to UK-PBC or GLOBE scores at 1-year, high NLR was able to identify those who are still at risk of poor outcome. The additional value of NLR supports efforts to further optimize model for decision making in clinical practice.

In conclusion, the UK-PBC and GLOBE scores seem to be good indicators of prognosis regardless of race or ethnicity. In addition, NLR may further help in the stratification of patients via capturing different perspectives of $\mathrm{PBC}$ by focusing on inflammation.

\section{CONFLICTS OF INTEREST}

No potential conflict of interest relevant to this article was reported.

\section{REFERENCES}

1. Hamlyn AN, Macklon AF, James O. Primary biliary cirrhosis: geographical clustering and symptomatic onset seasonality. Gut 1983;24:940-945.

2. European Association for the Study of the Liver. Electronic address eee, European Association for the Study of the L. EASL clinical practice guidelines: the diagnosis and management of patients with primary biliary cholangitis. J Hepatol 2017;67:145-172.

3. Parés A, Caballería L, Rodés J. Excellent long-term survival in patients with primary biliary cirrhosis and biochemical response to ursodeoxycholic acid. Gastroenterology 2006;130:715-720.

4. Corpechot C, Abenavoli L, Rabahi N, et al. Biochemical response to ursodeoxycholic acid and long-term prognosis in primary biliary cirrhosis. Hepatology 2008;48:871-877.

5. Corpechot C, Chazouillères O, Poupon R. Early primary biliary cirrhosis: biochemical response to treatment and prediction of longterm outcome. J Hepatol 2011;55:1361-1367.

6. Lammers WJ, Hirschfield GM, Corpechot C, et al. Development and validation of a scoring system to predict outcomes of patients with primary biliary cirrhosis receiving ursodeoxycholic acid therapy. Gastroenterology 2015;149:1804-1812.e4.

7. Carbone M, Sharp SJ, Flack S, et al. The UK-PBC risk scores: derivation and validation of a scoring system for long-term prediction of end-stage liver disease in primary biliary cholangitis. Hepatology 2016;63:930-950.
8. Muhmmed Suliman MA, Bahnacy Juma AA, Ali Almadhani AA, Pathare AV, Alkindi SS, Uwe Werner F. Predictive value of neutrophil to lymphocyte ratio in outcomes of patients with acute coronary syndrome. Arch Med Res 2010;41:618-622.

9. Liu H, Zhang H, Wan G, et al. Neutrophil-lymphocyte ratio: a novel predictor for short-term prognosis in acute-on-chronic hepatitis B liver failure. J Viral Hepat 2014;21:499-507.

10. Kuiper EM, Hansen BE, de Vries RA, et al. Improved prognosis of patients with primary biliary cirrhosis that have a biochemical response to ursodeoxycholic acid. Gastroenterology 2009;136:12811287.

11. Ripoll C, Groszmann R, Garcia-Tsao G, et al. Hepatic venous pressure gradient predicts clinical decompensation in patients with compensated cirrhosis. Gastroenterology 2007;133:481-488.

12. Umemura T, Joshita S, Sekiguchi T, et al. Serum wisteria floribunda agglutinin-positive Mac-2-binding protein level predicts liver fibrosis and prognosis in primary biliary cirrhosis. Am J Gastroenterol 2015;110:857-864.

13. Avanzas P, Quiles J, López de Sá E, et al. Neutrophil count and infarct size in patients with acute myocardial infarction. Int J Cardiol 2004;97:155-156.

14. Heagerty PJ, Lumley T, Pepe MS. Time-dependent ROC curves for censored survival data and a diagnostic marker. Biometrics 2000;56:337-344.

15. Papastergiou V, Tsochatzis EA, Rodriguez-Peralvarez M, et al. Biochemical criteria at 1 year are not robust indicators of response to ursodeoxycholic acid in early primary biliary cirrhosis: results from a 29-year cohort study. Aliment Pharmacol Ther 2013;38:1354-1364.

16. Yang F, Yang Y, Wang Q, et al. The risk predictive values of UKPBC and GLOBE scoring system in Chinese patients with primary biliary cholangitis: the additional effect of anti-gp210. Aliment Pharmacol Ther 2017;45:733-743.

17. Cheung KS, Seto WK, Fung J, Lai CL, Yuen MF. Prognostic factors for transplant-free survival and validation of prognostic models in Chinese patients with primary biliary cholangitis receiving ursodeoxycholic acid. Clin Transl Gastroenterol 2017;8:e100.

18. Webb GJ, Hirschfield GM. Primary biliary cholangitis in 2016: high-definition PBC: biology, models and therapeutic advances. Nat Rev Gastroenterol Hepatol 2017;14:76-78.

19. de Liso F, Matinato C, Ronchi M, Maiavacca R. The diagnostic accuracy of biomarkers for diagnosis of primary biliary cholangitis (PBC) in anti-mitochondrial antibody (AMA)-negative PBC patients: a review of literature. Clin Chem Lab Med 2017;56:25-31.

20. Imtiaz F, Shafique K, Mirza SS, Ayoob Z, Vart P, Rao S. Neutrophil lymphocyte ratio as a measure of systemic inflammation in prevalent chronic diseases in Asian population. Int Arch Med 2012;5:2.

21. Dogan I, Karaman K, Sonmez B, Celik S, Turker O. Relationship between serum neutrophil count and infarct size in patients with acute myocardial infarction. Nucl Med Commun 2009;30:797801. 
22. Martin HL, Ohara K, Kiberu A, Van Hagen T, Davidson A, Khattak MA. Prognostic value of systemic inflammation-based markers in advanced pancreatic cancer. Intern Med J 2014;44:676-682.

23. Kuo MT, Hu TH, Lu SN, et al. Neutrophil-to-lymphocyte ratio as a predictor of response to peginterferon plus ribavirin therapy for chronic hepatitis C. Dis Markers 2014;2014:462958.

24. Alkhouri N, Morris-Stiff G, Campbell C, et al. Neutrophil to lymphocyte ratio: a new marker for predicting steatohepatitis and fibrosis in patients with nonalcoholic fatty liver disease. Liver Int 2012;32:297-302.

25. Lin L, Piao M, Jiang X, et al. Does neutrophil-to-lymphocyte ratio predict 1-year mortality in patients with primary biliary cholangitis? Results from a retrospective study with validation cohort. BMJ Open 2017;7:e015304.

26. Li MX, Liu XM, Zhang XF, et al. Prognostic role of neutrophilto-lymphocyte ratio in colorectal cancer: a systematic review and meta-analysis. Int J Cancer 2014;134:2403-2413.

27. Cai YJ, Dong JJ, Dong JZ, et al. A nomogram for predicting prognostic value of inflammatory response biomarkers in decompensated cirrhotic patients without acute-on-chronic liver failure. Aliment Pharmacol Ther 2017;45:1413-1426. 